\title{
POROUS SETS AND QUASISYMMETRIC MAPS
}

\author{
JUSSI VÄISÄLÄ
}

\begin{abstract}
A set $A$ in $R^{n}$ is called porous if there is $\alpha>0$ such that every ball $\bar{B}(x, r)$ contains a point whose distance from $A$ is at least $\alpha r$. We show that porosity is preserved by quasisymmetric maps, in particular, by bilipschitz maps. Local versions are also given.
\end{abstract}

1. Introduction. We say that a set $A$ in the euclidean $n$-space $R^{n}$ is $\alpha$-porous in $R^{n}$, $0<\alpha \leqslant 1$, if each closed ball $\bar{B}(x, r)$ in $R^{n}$ contains a point $z$ such that the open ball $B(z, \alpha r)$ does not meet $A$. For example, $R^{p}$ is 1 -porous in $R^{p+1}$, and the Cantor middle-third set is $\frac{1}{4}$-porous in $R^{1}$.

Porous sets were considered by Granlund, Lindqvist and Martio [GLM, 4.15] in connection with harmonic measure. They also observed the close connection between porous sets (called thin in [GLM]) and uniform domains in the sense of [MS]. Indeed, it is easy to show that a closed set $A$ is porous in $R^{n}$ if and only if $R^{n+1} \backslash A$ is a uniform domain. Furthermore, Sarvas [Sa] proved that the branch set of a quasiregular map is always locally porous (see 4.4). The Hausdorff dimension of an $\alpha$-porous set is bounded by a number $c(\alpha, n)<n$; see [Sa, 3.2].

When this manuscript was completed, P. Mattila pointed out to the author that the term "porous set" has been used by E. P. Dolženko [Do] and others for sets which are weakly locally porous in the sense of 4.4.

If $f: R^{n} \rightarrow R^{n}$ is $K$-quasiconformal, it follows from the standard distortion theorems that $f$ maps every $\alpha$-porous set $A \subset R^{n}$ onto an $\alpha_{1}$-porous set with $\alpha_{1}=\alpha_{1}(\alpha, K, n)>0$. In this paper we consider the corresponding problem in the case where $f$ is only defined on $A$. We shall show (Theorem 4.2) that if $f: A \rightarrow R^{n}$ is $\eta$-quasisymmetric ( $\eta$-QS), then $f A$ is $\alpha_{1}$-porous with $\alpha_{1}=\alpha_{1}(\alpha, \eta)>0$. Observe that $\alpha_{1}$ does not depend on $n$. We recall [TV] that an embedding $f: A \rightarrow R^{n}$ is $\eta$-QS if $\eta:[0, \infty) \rightarrow[0, \infty)$ is a homeomorphism and if $|a-x| \leqslant t|b-x|$ implies $\mid f(a)-$ $f(x)|\leqslant \eta(t)| f(b)-f(x) \mid$ for all $a, b, x$ in $A$ and for all $t>0$. For example, if $f$ is L-bilipschitz, that is,

$$
|x-y| / L \leqslant|f(x)-f(y)| \leqslant L|x-y|
$$

for all $x, y \in A$, then $f$ is $\eta$-QS with $\eta(t)=L^{2} t$. A homeomorphism $f: R^{n} \rightarrow R^{n}$ is $\mathrm{QS}$ if and only if it is quasiconformal.

Received by the editors January 5, 1986.

1980 Mathematics Subject Classification (1985 Revision). Primary 30C60.

Key words and phrases. Porous, quasisymmetric, bilipschitz. 
Our basic result is Theorem 3.1, which states, roughly speaking, that QS maps preserve a property opposite to porosity. The QS invariance of porous sets and some related results are proved in $\$ 4$.

Our proof makes use of the topological degree of a map. P. Tukia pointed out to the author that by using normal families (his favorite tool) one can give a more elementary proof for a somewhat weaker result in which $\alpha_{1}$ also depends on $n$.

As a special case, we obtain corresponding results for bilipschitz maps. However, a direct proof for this case is somewhat simpler although it still makes use of degree theory. It also gives a better bound to $\alpha_{1}$.

I thank Olli Martio for inspiring discussions.

1.2. Remark. Since every $\eta$-QS map $f: A \rightarrow R^{n}$ has a unique extension to an $\eta$-QS map $\bar{f}: \bar{A} \rightarrow R^{n}$ [TV, 2.25], we could without loss of generality always assume that $A$ is closed. However, this would not simplify the proofs, and therefore we shall not give any topological restrictions to $A$.

1.3. Notation. We let $C A$ denote the complement $R^{n} \backslash A$ of a set $A \subset R^{n}$. The open $n$-ball with center $x$ and radius $r$ is written as $B^{n}(x, r)$ or $B(x, r)$, and we use the abbreviations $B^{n}(r)=B^{n}(0, r)$ and $B^{n}=B^{n}(0,1)$. If $A \subset R^{n}, B(A, r)$ denotes the $r$-neighborhood $A+B^{n}(r)$ of $A$.

2. The degree of a map. Let $D$ be a bounded domain in $R^{n}$, and let $f$ be a continuous map into $R^{n}$, defined in $\bar{D}$. The degree of $f$ is an integer $\mu(y, f, D)$ defined for every $y$ in $C f \partial D$. For the definition and the basic properties of the degree, we refer to [RR, II.2]. We recall that $y \mapsto \mu(y, f, D)$ is constant in each component of $C f \partial D$. We use the Alexander-Spanier cohomology theory with integral coefficients. We remark that [RR] uses a shift in dimension and writes $H^{n+1}(X, Y)$ for our $H^{n}(X, Y)$.

2.1. LemMA. Let $D \subset R^{n}$ be a bounded domain such that $C \bar{D}$ is connected. Suppose that $f: \bar{D} \rightarrow R^{n}$ is continuous and that $Y \subset R^{n}$ is a compact set containing $f \partial D$ such that the induced homomorphism $f_{1}^{*}: H^{n-1}(Y) \rightarrow H^{n-1}(\partial D)$ is not the zero map. Then there is a component $U$ of $C Y$ such that $\mu(y, f, D) \neq 0$ for $y \in U$.

Proof. We let $f_{1}, f_{2}, \ldots$ denote maps defined by $f$. Let $U_{1}, U_{2}, \ldots$ be the bounded components of $C Y$, let $U_{0}$ be their union, and set $X=U_{0} \cup Y$. Choose a closed ball $X_{1}$ containing $Y \cup f D$, and set $Y_{1}=X_{1} \backslash U_{0}$. Then $f$ defines a map $f_{2}$ : $(\bar{D}, \partial D) \rightarrow\left(X_{1}, Y_{1}\right)$. Furthermore, the inclusion $i:(X, Y) \rightarrow\left(X_{1}, Y_{1}\right)$ induces the following diagram for the cohomology sequences:

$$
\begin{aligned}
& \begin{array}{c}
H^{n-1}\left(X_{1}\right) \\
\end{array} \begin{array}{c}
H^{n-1}\left(Y_{1}\right) \\
\downarrow j^{*}
\end{array} \quad \rightarrow \quad \begin{array}{c}
H^{n}\left(X_{1}, Y_{1}\right) \\
\downarrow i^{*}
\end{array} \quad \rightarrow \quad H^{n}\left(X_{1}\right) \\
& H^{n-1}(X) \quad \rightarrow \quad H^{n-1}(Y) \quad \rightarrow \quad H^{n}(X, Y) \quad \rightarrow \quad H^{n}(X)
\end{aligned}
$$

Here $H^{n-1}\left(X_{1}\right)=H^{n-1}(X)=0$ by [RR, Theorem 2, p. 104], since $C X$ and $C X_{1}$ are connected. Moreover, $H^{n}\left(X_{1}\right)=H^{n}(X)=0$ by [RR, Theorem 1, p. 100]. Hence the middle horizontal arrows are bijective. Since $i^{*}$ is an excision isomorphism, $j^{*}$ is 
bijective. Consider the map $f_{3}: \partial D \rightarrow Y_{1}$. Since $f_{3}=j f_{1}, f_{3}^{*}: H^{n-1}\left(Y_{1}\right) \rightarrow H^{n-1}(\partial D)$ is not the zero map. Consider the diagram

$$
\begin{array}{ccccc} 
& H^{n-1}\left(Y_{1}\right) & \rightarrow & H^{n}\left(X_{1}, Y_{1}\right) \\
& \downarrow f_{3}^{*} & & \downarrow f_{2}^{*} \\
H^{n-1}(\partial D) \rightarrow & H^{n-1}(\partial D) & \stackrel{\delta}{\rightarrow} & H^{n}(\bar{D}, \partial D)
\end{array}
$$

Since $C \bar{D}$ is connected, $H^{n-1}(\bar{D})=0$. Hence $\delta$ is injective, which implies $f_{2}^{*} \neq 0$.

Set $Z_{j}=X_{1} \backslash U_{j}$. By [RR, p. 92], the inclusions $k_{j}:\left(X_{1}, Y_{1}\right) \rightarrow\left(X_{1}, Z_{j}\right)$ induce a representation of $H^{n}\left(X_{1}, Y_{1}\right)$ as the direct sum of the groups $H^{n}\left(X_{1}, Z_{j}\right)$. Then $f_{2}{ }^{*}$ is the sum of the homomorphisms $g_{j}^{*}: H^{n}\left(X_{1}, Z_{j}\right) \rightarrow H^{n}(\bar{D}, \partial D)$ where each $g_{j}$ is defined by $f$. Hence there is $j$ such that $g_{j}^{*} \neq 0$. Since $\left[X_{1}, Z_{j}, U_{j}\right]$ is a $(y, f, D)$ admissible frame in the sense of [RR, p. 121] for every $y \in U_{j}$, we have $\mu(y, f, D) \neq 0$ for all $y \in U_{j}$ [RR, p. 125].

3. The basic theorem. In this section we prove our basic result 3.1 , which states that if the complement of a set contains only small balls in a neighborhood of a point, then the QS image of the set has the same property. This can be considered as the QS version of the theorem on the invariance of domain. The bilipschitz version will be given in 3.17 .

3.1. TheOREM. Suppose that $f: A \rightarrow R^{n}$ is $\eta$-QS, where $A \subset \bar{B}^{n}, 0 \in A$, and $f(0)=0$. Suppose also that $0<\alpha \leqslant 1$ and that $\bar{B}^{n} \subset B(A, \alpha)$. Set $M=$ $\sup \{|f(x)|: x \in A\}$. Then there are positive numbers $\delta=\delta(\eta)$ and $\alpha^{\prime}=\alpha^{\prime}(\alpha, \eta)$ such that

(1) $\bar{B}^{n}(\delta M) \subset B\left(f A, \alpha^{\prime} M\right)$,

(2) $\alpha^{\prime}(\alpha, \eta) \rightarrow 0$ as $\alpha \rightarrow 0$.

3.2. OUtLINE OF THE PROOF. Since the QS image of a bounded set is bounded, $M<\infty$. Replacing $f$ by $f / M$, we may assume $M=1$. Since (1) is true for $\delta=1 / 2$, $\alpha^{\prime}=1$, it suffices to consider small values of $\alpha$. We always assume $\alpha \leqslant 1 / 6$. In the course of the proof, we shall give more restrictions of the type $\alpha \leqslant \alpha_{0}(\eta)$.

We shall construct a map $g: \bar{B}^{n} \rightarrow R^{n}$ such that $g \mid A$ approximates $f$ and $g$ is "roughly QS" although it is not usually injective. We show that $C g \partial B^{n}$ contains a ball $B^{n}(\delta)$ with $\delta=\delta(\eta)>0$ and that $\mu\left(y, g, B^{n}\right) \neq 0$ for $y \in B^{n}(\delta)$, which implies $B^{n}(\delta) \subset g B^{n}$. It follows that $B^{n}(\delta) \backslash g A$ can contain only small balls, and the same is then true for $B^{n}(\delta) \backslash f A$. The proof will be completed in 3.15 .

3.3. Construction of $g$. For $x \in R^{n}$ set $B_{x}=B^{n}(x, \alpha)$. Then $\left\{B_{a}: a \in A\right\}$ covers $\bar{B}^{n}$. Choose a partition of unity $\left(\phi_{a}\right)_{a \in A}$ in $\bar{B}^{n}$ subordinate to this cover. This means that for each $a \in A, \phi_{a}: \bar{B}^{n} \rightarrow[0,1]$ is continuous and spt $\phi_{a} \subset B_{a}$. Moreover, the family (spt $\left.\phi_{a}\right)_{a \in A}$ is locally finite, and $\sum_{a \in A} \phi_{a}(x)=1$ for all $x \in \bar{B}^{n}$. Define the map $g: \bar{B}^{n} \rightarrow R^{n}$ by

$$
g(x)=\sum_{a \in A} \phi_{a}(x) f(a) .
$$

Then $g(x)$ belongs to the convex hull of $f\left[A \cap B_{x}\right]$ for each $x \in \bar{B}^{n}$. Thus $g \bar{B}^{n} \subset \bar{B}^{n}$. 
3.4. $g \mid A$ approximates $f$. We show that

$$
|g(x)-f(x)| \leqslant 2 \eta(2 \alpha)
$$

for every $x \in A$. Choose $y \in \bar{B}^{n}$ with $|y-x| \geqslant 1$ and then $z \in A$ with $|z-y| \leqslant \alpha$. Then $|z-x| \geqslant 1 / 2$, and thus $|a-x| \leqslant 2 \alpha|z-x|$ for every $a \in B_{x}$. Hence

$$
|f(a)-f(x)| \leqslant \eta(2 \alpha)|f(z)-f(x)| \leqslant 2 \eta(2 \alpha)
$$

for $a \in B_{x}$. This implies

$$
|g(x)-f(x)| \leqslant \sum_{a \in A \cap B_{x}} \phi_{a}(x)|f(a)-f(x)| \leqslant 2 \eta(2 \alpha),
$$

and (3.5) is proved.

3.6. $g$ is roughly QS. We first show that for each pair of points $a, x \in \bar{B}^{n}$ there are points $a^{\prime}, x^{\prime} \in A$ such that

$$
\left|a-a^{\prime}\right| \leqslant 5 \alpha, \quad\left|x-x^{\prime}\right| \leqslant \alpha, \quad\left|a^{\prime}-x^{\prime}\right| \geqslant \alpha .
$$

There are points $a^{*}, x^{\prime}$ in $A$ such that $\left|a-a^{*}\right| \leqslant \alpha$ and $\left|x-x^{\prime}\right| \leqslant \alpha$. If $\left|x^{\prime}-a\right| \geqslant$ $2 \alpha$, we can choose $a^{\prime}=a^{*}$. Suppose that $\left|x^{\prime}-a\right|<2 \alpha$. Since $\alpha \leqslant 1 / 6$, there is $z \in \bar{B}^{n}$ with $\left|z-x^{\prime}\right|=2 \alpha$. Choose $a^{\prime} \in A \cap B_{z}$. Now

$$
\begin{gathered}
\left|a-a^{\prime}\right| \leqslant\left|a-x^{\prime}\right|+\left|x^{\prime}-z\right|+|z-a| \leqslant 5 \alpha, \\
\left|a^{\prime}-x^{\prime}\right| \geqslant\left|z-x^{\prime}\right|-\left|z-a^{\prime}\right| \geqslant \alpha,
\end{gathered}
$$

and thus (3.7) is true.

By rough quasisymmetry we mean the following: Setting

$$
\lambda=\lambda(\eta)=2+2 / \eta^{-1}\left(\frac{1}{4}\right)
$$

we prove that if $a, b, x \in \bar{B}^{n}$ with $|b-x| \geqslant \lambda \alpha$, then $g(b) \neq g(x)$ and

$$
\frac{|g(a)-g(x)|}{|g(b)-g(x)|} \leqslant M_{1} \eta\left(2 \frac{|a-x|+6 \alpha}{|b-x|}\right)
$$

where $M_{1}=M_{1}(\eta)$. Observe that $\lambda \geqslant 4$.

Choose $a^{\prime}, x^{\prime} \in A$ such that (3.7) is true. Then

$$
\left|g(a)-f\left(a^{\prime}\right)\right| \leqslant \sum_{y \in A \cap B_{a}} \phi_{y}(a)\left|f(y)-f\left(a^{\prime}\right)\right| .
$$

For each $y \in A \cap B_{a}$ we have $\left|y-a^{\prime}\right| \leqslant 6\left|x^{\prime}-a^{\prime}\right|$, and hence

$$
\left|g(a)-f\left(a^{\prime}\right)\right| \leqslant \eta(6)\left|f\left(a^{\prime}\right)-f\left(x^{\prime}\right)\right| .
$$

A similar argument gives

$$
\left|g(x)-f\left(x^{\prime}\right)\right| \leqslant \eta(2)\left|f\left(a^{\prime}\right)-f\left(x^{\prime}\right)\right|,
$$

and we obtain

$$
\begin{aligned}
|g(a)-g(x)| & \leqslant(1+\eta(2)+\eta(6))\left|f\left(a^{\prime}\right)-f\left(x^{\prime}\right)\right| \\
& \leqslant 3 \eta(6)\left|f\left(a^{\prime}\right)-f\left(x^{\prime}\right)\right| .
\end{aligned}
$$


Choose $b^{\prime} \in A \cap B_{b}$. Then

$$
|g(b)-g(x)| \geqslant\left|f\left(b^{\prime}\right)-f\left(x^{\prime}\right)\right|-\left|g(b)-f\left(b^{\prime}\right)\right|-\left|g(x)-f\left(x^{\prime}\right)\right| .
$$

For every $y \in A \cap B_{b}$ we have

$$
\left|y-b^{\prime}\right| \leqslant 2 \alpha \leqslant \frac{2}{\lambda-2}\left|x^{\prime}-b^{\prime}\right|,
$$

and the same estimate holds for $\left|y-x^{\prime}\right|, y \in A \cap B_{x}$. Arguing as above yields

$$
|g(b)-g(x)| \geqslant\left(1-2 \eta\left(\frac{2}{\lambda-2}\right)\right)\left|f\left(x^{\prime}\right)-f\left(b^{\prime}\right)\right|=\frac{\left|f\left(x^{\prime}\right)-f\left(b^{\prime}\right)\right|}{2} .
$$

Since $\lambda \geqslant 4$, we have $\left|b^{\prime}-x^{\prime}\right| \geqslant|b-x| / 2$. Hence (3.9) and (3.10) give (3.8) with $M_{1}=6 \eta(6)$.

3.11. Estimate for $d\left(g \partial B^{n}, g(0)\right)$. We next show that for small $\alpha, d\left(g \partial B^{n}, g(0)\right)$ is bounded from below by a positive constant $r=r(\eta)$. We make the following new restrictions for $\alpha$ :

$$
2 \lambda \alpha \leqslant 1, \quad 8 \eta(2 \alpha) \leqslant 1 .
$$

Suppose that $x \in \partial B^{n}$ and set $y=g(x)$. Choose $x_{0} \in A$ with $\left|f\left(x_{0}\right)\right| \geqslant 3 / 4$ and set $y_{0}=g\left(x_{0}\right)$. Since $|x|=1>\lambda \alpha$, we can apply (3.8) with the substitution $(x, a, b)$ $\mapsto\left(0, x_{0}, x\right)$ and obtain

$$
\frac{\left|y_{0}-g(0)\right|}{|y-g(0)|} \leqslant M_{1} \eta\left(2\left|x_{0}\right|+12 \alpha\right) \leqslant M_{1} \eta(4) .
$$

On the other hand, (3.5) gives

$$
\left|y_{0}-g(0)\right| \geqslant\left|f\left(x_{0}\right)\right|-4 \eta(2 \alpha) \geqslant 1 / 4 \text {. }
$$

Consequently,

$$
|y-g(0)| \geqslant 1 / 4 M_{1} \eta(4)=r(\eta) .
$$

3.13. The degree of $g$. We want to show that for small $\alpha, \mu\left(y, g, B^{n}\right) \neq 0$ for $y \in B^{n}(g(0), r)$. Set

$$
Y=g \partial B^{n}, \quad Y(t)=\bar{B}(Y, t), \quad Q(y, t)=\partial B^{n} \cap g^{-1}[\bar{B}(y, t) \cap Y],
$$

where $t>0$ and $y \in Y$.

We shall first find an upper bound for $d(Q(y, t))$. Let $x, b \in Q(y, t)$. If $|b-x| \geqslant \lambda \alpha$, we can apply (3.8) with $a=0$, and obtain

$$
\frac{|g(0)-g(x)|}{|g(b)-g(x)|} \leqslant M_{1} \eta\left(\frac{4}{|b-x|}\right) \text {. }
$$

On the other hand, 3.11 gives $|g(0)-g(x)| \geqslant r$. Since $|g(b)-g(x)| \leqslant 2 t$, we get

$$
|b-x| \leqslant \frac{4}{\eta^{-1}\left(r / 2 M_{1} t\right)}=\psi(t, \eta) \rightarrow 0
$$

as $t \rightarrow 0$. Hence

$$
d(Q(y, t)) \leqslant \max (\lambda \alpha, \psi(t, \eta)) .
$$


Choose $t_{0}>0$ such that $\psi(t, \eta) \leqslant 1$ for $t \leqslant t_{0}$. By (3.12) we obtain $d(Q(y, t)) \leqslant 1$ for $t \leqslant t_{0}$.

Set $t=t_{0} / 2$ and cover $Y(t)$ with a finite number of balls $B\left(y_{1}, t_{0}\right), \ldots, B\left(y_{k}, t_{0}\right)$ with centers $y_{j} \in Y$. For each $j$ choose $x_{j} \in f^{-1}\left(y_{j}\right) \cap \partial B^{n}$. Set $F_{j}=Y(t) \backslash B\left(y_{j}, t_{0}\right)$ and define $h: Y(t) \rightarrow R^{n}$ by

$$
h(y)=\sum_{j=1}^{k} d\left(y, F_{j}\right) x_{j} .
$$

For every $y \in Y(t)$ there is at least one $j$ with $d\left(y, F_{j}\right) \neq 0$. For each such $j$, we have $y_{j} \in B\left(y, t_{0}\right)$ and hence $x_{j} \in Q\left(y, t_{0}\right)$. Since $d\left(Q\left(y, t_{0}\right)\right) \leqslant 1, Q\left(y, t_{0}\right)$ is contained in an open hemisphere $H(y)$ of $\partial B^{n}$, which implies $h(y) \neq 0$. We can thus define $u: Y(t) \rightarrow \partial B^{n}$ by $u(y)=h(y) /|h(y)|$. Then $u(y) \in H(y)$. Since $x \in Q\left(g(x), t_{0}\right)$ for every $x \in \partial B^{n},|u(g(x))-x|<2$ for all $x \in \partial B^{n}$. This implies that the map $u g_{1}: \partial B^{n} \rightarrow \partial B^{n}$ is homotopic to the identity map of $\partial B^{n}$; here $g_{1}: \partial B^{n} \rightarrow Y(t)$ is the map defined by $g$. Consequently, the induced homomorphism $g_{1}^{*}: H^{n-1}(Y(t)) \rightarrow H^{n-1}\left(\partial B^{n}\right)=Z$ is not the zero map. By Lemma 2.1, there is a component $U$ of $C Y(t)$ such that $\mu\left(y, g, B^{n}\right) \neq 0$ for $y \in U$. Hence $U \subset g B^{n}$.

We want to show that $g(0) \in U$ for small $\alpha$. Choose $x_{0} \in B^{n}$ such that $g\left(x_{0}\right) \in U$. If $x_{0}=0$, there is nothing to prove. If $x_{0} \neq 0$, let $J$ be the line segment with endpoints 0 and $x_{0}$. It suffices to show that $Y \cap g J=\varnothing$. Since $g(b) \neq g(x)$ whenever $|b-x| \geqslant \lambda \alpha$, it suffices to show $\left|x_{0}\right| \leqslant 1-\lambda \alpha$. Suppose that $\left|x_{0}\right|>1-$ $\lambda \alpha$. By (3.12) we have $\lambda \alpha \leqslant 1 / 2$, which implies $\left|x_{0}\right|>\lambda \alpha$. Setting $x_{1}=x_{0} /\left|x_{0}\right|$, we can thus apply (3.8) with $(x, a, b) \mapsto\left(x_{0}, x_{1}, 0\right)$ and obtain

$$
\frac{\left|g\left(x_{1}\right)-g\left(x_{0}\right)\right|}{\left|g(0)-g\left(x_{0}\right)\right|} \leqslant M_{1} \eta\left(2 \frac{\left|x_{1}-x_{0}\right|+6 \alpha}{\left|x_{0}\right|}\right) \leqslant M_{1} \eta\left(4\left(\left|x_{1}-x_{0}\right|+6 \alpha\right)\right) \text {. }
$$

Since $g \bar{B}^{n} \subset \bar{B}^{n}$, the left side is at least $t / 2$. Hence

$$
\left|x_{1}-x_{0}\right| \geqslant \frac{\eta^{-1}\left(t / 2 M_{1}\right)}{4}-6 \alpha .
$$

We make the new restriction

$$
\alpha \max (6, \lambda) \leqslant \frac{\eta^{-1}\left(t / 2 M_{1}\right)}{8} .
$$

Then $1-\left|x_{0}\right|=\left|x_{1}-x_{0}\right| \geqslant \lambda \alpha$, which gives a contradiction. Hence $g(0) \in U$. By 3.11, $U$ contains the ball $B(g(0), r)$. Consequently,

$$
B(g(0), r) \subset g B^{n} .
$$

3.15. COMPLETION OF THE PROOF. We show that Theorem 3.1 is true with $\delta=r / 2$ and with a suitable $\alpha^{\prime}$. Let $y \in \bar{B}^{n}(\delta)$. Since $f(0)=0$, (3.5) implies

$$
|y-g(0)| \leqslant r / 2+2 \eta(2 \alpha) \leqslant r
$$

as soon as $\alpha$ satisfies the new restriction $4 \eta(2 \alpha) \leqslant r$. By (3.14), this yields $y \in g \bar{B}^{n}$. Choose $x \in \bar{B}^{n}$ with $y=g(x)$ and then $x^{\prime} \in A$ and $x_{1} \in \bar{B}^{n}$ with $\left|x^{\prime}-x\right|<\alpha$ and 
$\left|x_{1}-x\right|=1$. By (3.12) we have $\left|x_{1}-x\right|>\lambda \alpha$. Hence we can apply (3.8) with $(x, a, b) \mapsto\left(x, x^{\prime}, x_{1}\right)$ and obtain

$$
\frac{\left|g\left(x^{\prime}\right)-y\right|}{\left|g\left(x_{1}\right)-y\right|} \leqslant M_{1} \eta\left(2\left(\left|x^{\prime}-x\right|+6 \alpha\right)\right) \leqslant M_{1} \eta(14 \alpha) .
$$

Thus $\left|g\left(\alpha^{\prime}\right)-y\right|<2 M_{1} \eta(14 \alpha)$. By (3.5) this gives

$$
\left|f\left(x^{\prime}\right)-y\right|<2 M_{1} \eta(14 \alpha)+2 \eta(2 \alpha)=\alpha^{\prime}(\alpha, \eta),
$$

and Theorem 3.1 is proved.

3.16. The bilipschitz case. Suppose that in Theorem $3.1 f: A \rightarrow R^{n}$ is $L$-bilipschitz. Then $f$ is $\eta$-QS with $\eta(t)=L^{2} t$, and 3.1 gives $\delta$ and $\alpha^{\prime}$ depending on $L$ and $\alpha$. However, in this special case one can somewhat simplify the proof and also obtain a sharp bound for $\boldsymbol{\alpha}^{\prime}$. First, we apply Kirszbraun's theorem [Fe, 2.10.43] and extend $f$ to an $L$-lipschitz map, still denoted by $f: \bar{B}^{n} \rightarrow R^{n}$. This map it not necessarily injective, but it satisfies the rough bilipschitz condition

$$
|x-y| / L-2(L+1) \alpha \leqslant|f(x)-f(y)| \leqslant L|x-y|
$$

for all $x, y \in \bar{B}^{n}$. This extension replaces the map $g$ of 3.3. If $4 L(L+1) \alpha \leqslant 1$, $f \partial B^{n}$ does not meet the ball $B^{n}(1 / 2 L)$. As in 3.13 , we can use degree theory to show that for small $\alpha, \bar{B}^{n}(1 / 2 L) \subset f \bar{B}^{n}$. Indeed, this is true if $16 L^{3}(L+1) \alpha \leqslant 1$. Let $y \in \bar{B}^{n}(1 / 2 L)$. Choose $x \in \bar{B}^{n}$ and $x^{\prime} \in A$ with $y=f(x)$ and $\left|x^{\prime}-x\right|<\alpha$. Then $\left|y-f\left(x^{\prime}\right)\right|<L \alpha$, and we obtain the following result.

3.17. ThEOREM. Suppose that $f: A \rightarrow R^{n}$ is L-bilipschitz, where $A \subset \bar{B}^{n}, 0 \in A$, and $f(0)=0$. Suppose also that $0<\alpha \leqslant 1 / 16 L^{3}(L+1)$ and that $\bar{B}^{n} \subset B(A, \alpha)$. Then $\bar{B}^{n}(1 / 2 L) \subset B(f A, L \alpha)$.

3.18. Remark. The bound $\alpha^{\prime}=L \alpha$ in 3.17 is best possible. To see this, take any $\beta<\alpha$ and then $A=\bar{B}^{n} \backslash B^{n}\left(\beta e_{1}, \beta\right), f(\alpha)=L x$. Then $\bar{B}^{n}(1 / 2 L) \not \subset B(f A, L \beta)$ provided that $2 L^{2} \beta \leqslant 1$.

On the other hand, the radius $1 / 2 L$ can be replaced by the number $\delta(L)=1 / L$ $-2(L+1) \alpha$, which tends to $1 / L$ as $\alpha \rightarrow 0$.

4. Applications. We first prove the QS invariance of porous sets. Then we give two local versions and consider the bilipschitz case in which we also give a "whole space" version of 3.17 .

4.1. Porous sets. We recall the definition given in the introduction. Let $0<\alpha \leqslant 1$. A set $A \subset R^{n}$ is $\alpha$-porous in $R^{n}$ if every ball $\bar{B}(x, r)$ contains a point $z$ such that $B(z, \alpha r) \cap A=\varnothing$. In other words, $\bar{B}(x, r) \not \subset B(A, \alpha r)$. Still in other words, $\sup \{d(z, A) / r:|z-x| \leqslant r\} \geqslant \alpha$ for every $x \in R^{n}$ and $r>0$.

Although $x$ is an arbitrary point in $R^{n}$, it is easy to see that if the condition is satisfied for all $x \in A$, then $A$ is $\alpha / 2$-porous.

O. Martio observed that porosity is equivalent to the following condition: There is $c \geqslant 1$ such that $A \subset B(\partial B(A, t), c t)$.

From the compactness of $\bar{B}(x, r)$ it easily follows that for each porous set $A$ there is a maximal $\alpha \leqslant 1$ such that $A$ is $\alpha$-porous; this number might be called the porosity constant of $A$. 
4.2. TheOREM. Suppose that $A$ is $\alpha$-porous in $R^{n}$ and that $f: A \rightarrow R^{n}$ is $\eta$-QS. Then $f A$ is $\alpha_{1}$-porous with $\alpha_{1}=\alpha_{1}(\alpha, \eta)$.

Proof. Suppose that $0<\beta<1 / 4$ and that $A^{\prime}=f A$ is not $\beta$-porous. From 4.1 it follows that there is $y_{0} \in A^{\prime}$ and $r>0$ such that for every $z \in \bar{B}\left(y_{0}, r\right)$, the ball $B(z, 2 \beta r)$ meets $A^{\prime}$. Hence $B(z, 4 \beta r)$ meets $A^{\prime} \cap \bar{B}\left(y_{0}, r\right)$ for every $z \in \bar{B}\left(y_{0}, r\right)$. Let $g: A^{\prime} \rightarrow A$ be the inverse map of $f$ and set $g_{1}(y)=g\left(y_{0}+r y\right)-g\left(y_{0}\right)$. Then $g$ and $g_{1}$ are $\eta^{\prime}$-QS with $\eta^{\prime}(t)=\eta^{-1}\left(t^{-1}\right)^{-1}$. We consider $g_{1}$ as a map of $A_{1}=\bar{B}^{n} \cap$ $r^{-1}\left(A^{\prime}-y_{0}\right)$. Then we can apply Theorem 3.1 with the substitution $(A, f, \eta, \alpha) \mapsto$ $\left(A_{1}, g_{1}, \eta^{\prime}, 4 \beta\right)$. We obtain the numbers $\delta=\delta\left(\eta^{\prime}\right)$ and $\alpha^{\prime}=\alpha^{\prime}\left(4 \beta, \eta^{\prime}\right)$. Condition (1) of 3.1 implies that $g_{1} A_{1}$ is not $\alpha^{\prime} / \delta$-porous. Since $g_{1} A_{1}+g\left(y_{0}\right) \subset A, \alpha<\alpha^{\prime} / \delta$. Since $\alpha^{\prime} \rightarrow 0$ as $\beta \rightarrow 0$, we obtain a lower bound for $\beta$ of the type $\beta>\alpha_{1}(\alpha, \eta)>0$.

4.3. Remark. If $p<n$, every set $A \subset R^{p}$ is 1-porous in $R^{n}$. Consequently, if $f$ : $A \rightarrow R^{n}$ is $\eta$-QS, then $f A$ is $\alpha_{1}$-porous with $\alpha_{1}=\alpha_{1}(\eta)$. In particular, the Hausdorff dimension of $f A$ is bounded by a number $c(n, \eta)<n$. This was proved in [Vä, 4.1] in the special case where $A$ is open in $R^{p}$.

4.4. Local versions. We say that a set $A \subset R^{n}$ is weakly locally $\alpha$-porous at a point $x \in \bar{A}$ if there are arbitrarily small numbers $r>0$ such that $\bar{B}(x, r) \not \subset B(A, \alpha r)$. If there is $r_{0}>0$ such that this condition holds for all positive $r \leqslant r_{0}, A$ is locally $\alpha$-porous at $x$. These notions were suggested by O. Martio. He also observed that a weakly locally porous set cannot have points of density and therefore has $n$-measure zero.

We omit the proof of the following result, since it is similar to the proof of 4.2.

4.5. THEOREM. Suppose that $A \subset R^{n}$ is locally $\alpha$-porous or locally weakly $\alpha$-porous at $x \in A$ and that $f: A \rightarrow R^{n}$ is $\eta$-QS. Then $f A$ is locally $\alpha_{1}$-porous or locally weakly $\alpha_{1}$-porous, respectively, at $f(x)$ with $\alpha_{1}=\alpha_{1}(\alpha, \eta)$.

4.6. The bilipschitz case. Suppose that $A \subset R^{n}$ and that $f: A \rightarrow R^{n}$ is $L$-bilipschitz. If $A$ is $\alpha$-porous, then 4.2 implies that $f A$ is $\alpha_{1}$-porous with $\alpha_{1}=\alpha_{1}(\alpha, L)$. Replacing 3.1 by 3.17 in the proof of 4.2 , we easily see that one can choose $\alpha_{1}=\alpha / 8 L^{2}$. Similarly, the bilipschitz version of 4.5 is true with $\alpha_{1}=\alpha / 4 L^{2}$.

We finally give a bilipschitz result whose direct QS analogue is false. We say that a set $A \subset R^{n}$ is a $q$-net in $R^{n}, q>0$, if $B(A, q)=R^{n}$. For example, the set $Z$ of integers is a 1 -net in $R^{1}$. The map $f: R^{1} \rightarrow R^{1}, f(x)=x^{3}$ is QS but $f Z$ is not a $q$-net for any $q>0$. Hence the net property is not a QS invariant. However, it is a bilipschitz invariant:

4.7. TheOREM. Suppose that $A$ is a q-net in $R^{n}$ and that $f: A \rightarrow R^{n}$ is L-bilipschitz. Then $f A$ is an Lq-net in $R^{n}$.

Proof. We may assume that $0 \in A$ and that $f(0)=0$. Let $M>1$, set $A_{M}=\bar{B}^{n}$ $\cap M^{-1} A$, and define $f_{M}: A_{M} \rightarrow R^{n}$ by $f_{M}(x)=M^{-1} f(M x)$. Then $f$ is $L$-bilipschitz. Apply 3.17 with the substitution $(A, f, \alpha) \mapsto\left(A_{M}, f_{M}, q / M\right)$. This is possible if $M \geqslant 16 q L^{3}(L+1)$. We obtain $\bar{B}^{n}(1 / 2 L) \subset B\left(f_{M} A_{M}, L q / M\right)$, which yields $\bar{B}^{n}(M / 2 L) \subset B^{n}(f A, L q)$. Letting $M \rightarrow \infty$ proves the theorem. 


\section{REFERENCES}

[Do] E. P. Dolženko, Boundary properties of arbitrary functions, Izv. Akad. Nauk. SSSR Ser. Mat. 31 (1967), 3-14. (Russian)

[GLM] S. Granlund, P. Lindqvist and O. Martio, F-harmonic measure in space, Ann. Acad. Sci. Fenn. Ser. AI Math. 7 (1982), 233-247.

[MS] O. Martio and J. Sarvas. Injectivity theorems in plane and in space, Ann. Acad. Sci. Fenn. Ser. AI Math. 4 (1979), 383-401.

[Sa] J. Sarvas, The Hausdorff dimension of the branch set of a quasiregular mapping, Ann. Acad. Sci. Fenn. Ser. AI Math. 1 (1975), 297-307.

[TV] P. Tukia and J. Väisälä, Quasisymmetric embeddings of metric spaces, Ann. Acad. Sci. Fenn. Ser. AI Math. 5 (1980), 97-114.

[RR] T. Rado and P. V. Reichelderfer, Continuous transformations in analysis, Springer-Verlag, 1955.

[Fe] H. Federer, Geometric measure theory, Springer-Verlag, 1969.

[Vä] J. Väisälä, Quasi-symmetric embeddings in euclidean spaces, Trans. Amer. Math. Soc. 264 (1981), 191-204.

Department of Mathematics, University of Helsinki, Helsinki, Finland 